\title{
Recruitment of Grb2 and SHIP1 by the ITT-like motif of TIGIT suppresses granule polarization and cytotoxicity of NK cells
}

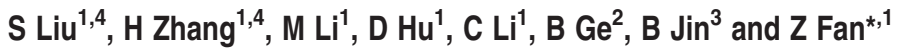

Activating and inhibitory receptors control natural killer (NK) cell activity. T-cell immunoglobulin and ITIM (immunoreceptor tyrosine-based inhibition motif) domain (TIGIT) was recently identified as a new inhibitory receptor on T and NK cells that suppressed their effector functions. TIGIT harbors the immunoreceptor tail tyrosine (ITT)-like and ITIM motifs in its cytoplasmic tail. However, how its ITT-like motif functions in TIGIT-mediated negative signaling is still unclear. Here, we show that TIGIT/PVR (poliovirus receptor) engagement disrupts granule polarization leading to loss of killing activity of NK cells. The ITT-like motif of TIGIT has a major role in its negative signaling. After TIGIT/PVR ligation, the ITT-like motif is phosphorylated at Tyr225 and binds to cytosolic adapter Grb2, which can recruit SHIP1 to prematurely terminate phosphatidylinositol 3-kinase (PI3K) and MAPK signaling, leading to downregulation of NK cell function. In support of this, Tyr225 or Asn227 mutation leads to restoration of TIGIT/PVR-mediated cytotoxicity, and SHIP1 silencing can dramatically abolish TIGIT/PVR-mediated killing inhibition.

Cell Death and Differentiation (2013) 20, 456-464; doi:10.1038/cdd.2012.141; published online 16 November 2012

Natural killer (NK) cells are key effectors in innate immunity which have an important role in the defense against tumors and viruses. ${ }^{1}$ NK cells can directly kill transformed or virusinfected cells very effectively. ${ }^{2-4}$ Meanwhile, normal cells are kept away from their cytotoxicity. Therefore, the discrimination between 'self' normal cells and 'nonself' abnormal cells has to be precisely recognized by NK cells. ${ }^{5-7} \mathrm{~A}$ very large repertoire of receptors, both activating and inhibitory, is proved to be critical in NK cell function. The best-known inhibitory receptors of NK cells are the killer-cell immunoglobulin-like receptor family, whose physical ligands are MHC-I molecules that are expressed on self normal cells to protect them from NK cell lysis. ${ }^{8}$ Other non-MHC-I inhibitory receptors, which do not associate with MHC-I molecules, are also expressed on NK cells. ${ }^{9}$ However, their physiological and pathological significances have not been defined yet.

T-cell immunoglobulin and ITIM domain (TIGIT) was recently identified as an inhibitory receptor that is expressed mainly on NK cells, activated CD4 and CD8 T cells. ${ }^{10-12}$ TIGIT harbors one extracellular immunoglobulin domain, a type 1 transmembrane region, and an immunoglobulin tail tyrosine (ITT)-like phosphorylation motif followed by an ITIM (immunoreceptor tyrosine-based inhibition motif) of the cytoplasmic tail. ${ }^{13}$ The physical ligands of TIGIT were identified as the poliovirus receptor (PVR, or CD155) and the PVRL2 (Nectin2, or CD112). ${ }^{10,12}$ TIGIT can bind to PVR of human dendritic cells to enhance interleukin 10 (IL-10) production, which inhibits T-cell activation. ${ }^{10}$ Kuchroo et al. ${ }^{14}$ showed that TIGIT harbors a T-cell-intrinsic inhibitory function to suppress T-cell activation.
Moreover, TIGIT can inhibit NK cell cytolysis through engagement with PVR or PVRL2. ${ }^{11}$ TIGIT-deficient mice are more susceptible to autoimmune diseases. ${ }^{14,15}$ However, the inhibitory mechanism mediated by TIGIT has not been elucidated.

TIGIT contains a classical ITIM motif, which recruits either Src homology $(\mathrm{SH}) 2$ domain-containing protein tyrosine phosphatases SHP1 and SHP2 or the inositol phosphatases SHIP1 and SHIP2 to mediate a negative signaling. ${ }^{16}$ Interestingly, TIGIT also has an ITT-like motif nearby the ITIM motif in the cytoplasmic segment, characterized by $\mathrm{YxN}$ consensus residues. ITT-like modules provide a basis for recruiting the cytosolic adapter Grb2 and/or the class IA phosphatidylinositol 3-kinase (PI3K). More than 30 associated proteins with Grb2 have been identified in B cells. ${ }^{17} \mathrm{~A}$ report showed that the ITT motif in the cytoplasmic regions of membrane-bound IgG and IgE initiates ITAM (immunoreceptor tyrosine-based activation motif)-induced signaling and B-cell proliferation. ${ }^{18}$ However, how ITT-like motifs function in NK or T cells is still unclear. Here we found that TIGIT associates with Grb2 and SHIP1 mainly through its ITT-like motif to induce a negative signaling, leading to the downregulation of NK cell effector function.

\section{Results}

TIGIT is clustered to the immunological synapse and disrupts granule polarization and cytotoxicity of NK cells once engaged with PVR. We used the NK cell line YTS cells as effector killers and the 721.221 cells as targets, which execute a restricted killing mainly through the

${ }^{1}$ CAS Key Laboratory of Infection and Immunity, Institute of Biophysics, Chinese Academy of Sciences, Beijing, China; ${ }^{2}$ Translational and Clinical Center of Shanghai Pulmonary Hospital, School of Medicine, Tongji University, Shanghai, China and ${ }^{3}$ Department of Immunology, the Fourth Military Medical University, Xi'an, China ${ }^{*}$ Corresponding author: Z Fan, CAS Key Laboratory of Infection and Immunity, Institute of Biophysics, Chinese Academy of Sciences, 15 Datun Road, Beijing 100101, China. Tel: +86 10 64888457; Fax: +86 10 64871293; E-mail: fanz@moon.ibp.ac.cn

${ }^{4}$ These authors contributed equally to this work.

Keywords: NK cells; TIGIT; Grb2; SHIP1; PI3K signaling

Abbreviations: NK, natural killer; TIGIT, T-cell immunoglobulin and ITIM domain; PVR, poliovirus receptor; ITT, immunoreceptor tail tyrosine

Received 08.5.12; revised 06.10.12; accepted 09.10.12; Edited by RA Knight; published online 16.11 .12 

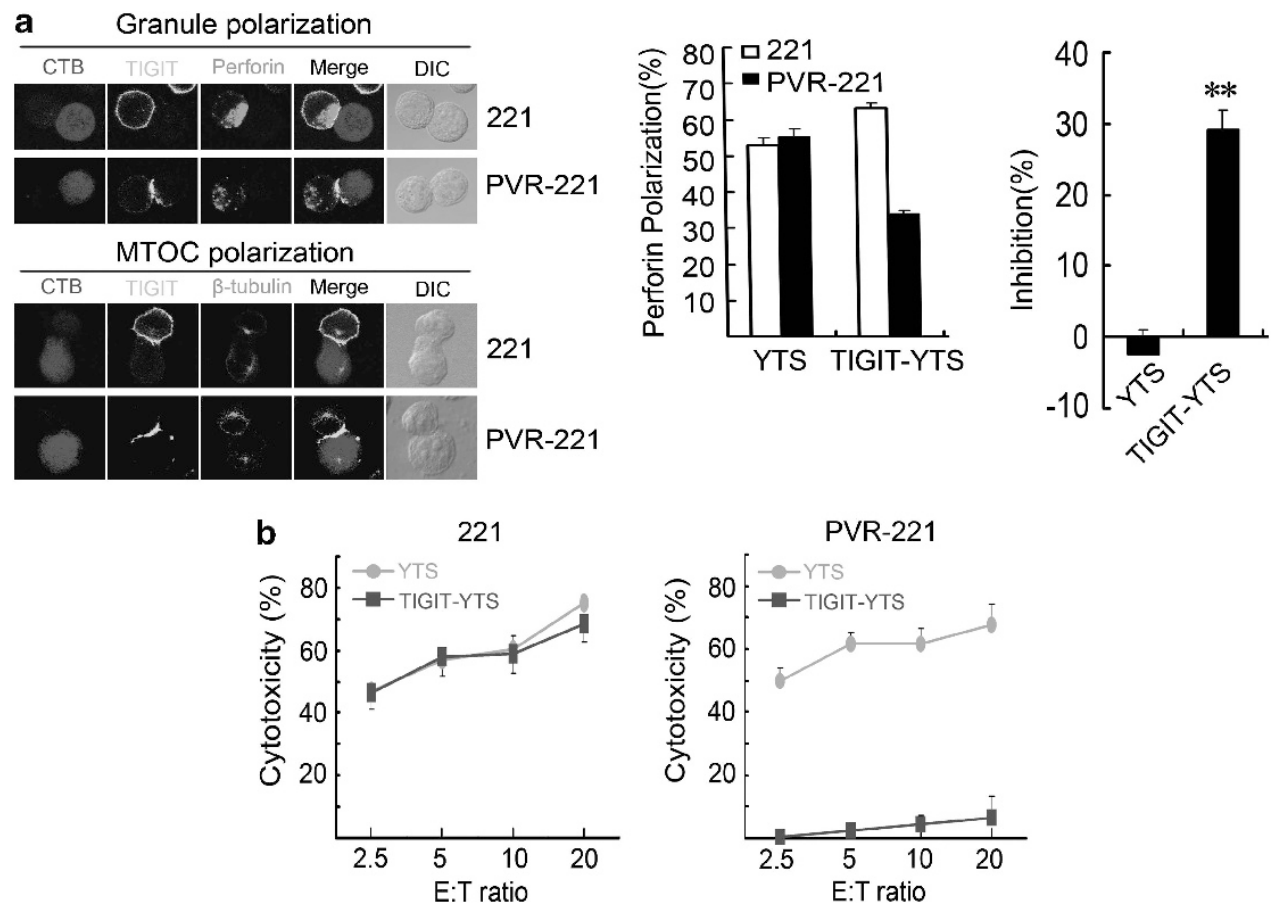

Figure 1 TIGIT/PVR engagement disrupts granule polarization and cytotoxicity of YTS cells. (a) TIGIT/PVR engagement disrupts granule and MTOC polarization. PVR221 or 221 target cells were labeled by CellTracker Blue and then incubated with TIGIT-YTS or YTS cells at $37^{\circ} \mathrm{C}$ for 30 min. Treated cells were stained with anti-Flag and antiperforin antibody (upper panel) or anti- $\beta$-tubulin antibody (lower panel) followed by confocal microscopy. Blue fluorescence for target cells is shown in the first lane, green for TIGIT in the second lane, red for perforin or $\beta$-tubulin in the third lane, the merged image in the fourth lane and differential interference control (DIC) in the last. Granule polarization (middle) was counted at least 100 conjugates in each experiment. Inhibition rates (right) of YTS and TIGIT-YTS were calculated as the polarization rate against 221 cells minus the polarization rate against PVR-221 cells. These data are representative of at least six separate experiments shown as means \pm S.D. The Student's $t$ test was used for statistical analysis. ${ }^{\star \star} P<0.01$. CTB: CellTracker Blue; 221: 721.221 cells. (b) TIGIT/PVR engagement suppresses YTS cell-mediated cytolysis. PVR-221 (right) or 221 (left) target cells were labeled with ${ }^{51} \mathrm{Cr}$ and incubated with TIGIT-YTS or YTS cells at the indicated E:T ratios followed by a ${ }^{51} \mathrm{Cr}$-release assay. Data are representative of at least three independent experiments as means \pm S.D. The color representation of this figure is available at the Cell Death and Differentiation Journal online

interaction between the 2B4 receptor on YTS cells and its ligand CD48 on the target cells. ${ }^{11}$ Additionally, Stanietsky et al. ${ }^{11}$ showed that CD226 and CD96 are expressed on YTS cells, but they are unable to enhance the killing ability mediated by PVR for an unknown reason. Actually, YTS cells did not express TIGIT, and 721.221 cells did not have PVR. YTS cells stably expressing Flag-tagged TIGIT (named TIGIT-YTS) and 721.221 cells with PVR expression (named PVR-221) were established (Supplementary Figure S1A). PVR (also called as Necl-5) is a member of the Nectin-like family, which belongs to adhesion molecules. ${ }^{19,20}$ To determine whether TIGIT/PVR ligation participates in the adhesion between NK and the target cells, we performed a conjugation assay. We found that the TIGIT/PVR engagement did not affect the conjugation rates between YTS and 221 cells (Supplementary Figure S1B).

To evaluate whether TIGIT/PVR ligation affects granule polarization, we incubated TIGIT-YTS cells with CellTracker Blue-labeled PVR-221 cells followed by staining with perforin and TIGIT. Without PVR, TIGIT was evenly stained on cell membrane and granules polarized toward the immunological synapse (IS) (Figure 1a, upper panel). Once engaged with PVR, TIGIT was clustered to the IS, whereas granules randomly distributed and failed to directly polarize to the IS. These data are representative of at least six independent experiments and the statistical analysis is shown in the right panel of Figure 1a. The microtubule organization center (MTOC) did not move toward the IS after TIGIT/PVR ligation, while MTOC could polarize to the IS without TIGIT/PVR engagement (Figure 1a, lower panel). Moreover, TIGIT/PVR ligation almost completely blocked NK cell-mediated cytolysis (Figure 1b), which is in agreement with a recent report. ${ }^{11}$

TIGIT is phosphorylated at its cytoplasmic tail after its ligation with PVR. It was shown that the first step of the inhibitory signaling pathway is phosphorylation of ITIM in the inhibitory receptors. ${ }^{8}$ To test whether TIGIT is phosphorylated, we incubated TIGIT-YTS with PVR-221 cells and immunoprecipitated TIGIT-Flag with anti-Flag antibody followed by immunoblotting with anti-phosphotyrosine antibody. With TIGIT/PVR engagement, TIGIT was tyrosine phosphorylated (Figure 2a). TIGIT has two tyrosines, Tyr225 in the ITT-like motif and Tyr231 in the ITIM motif, of its cytoplasmic segment. To further determine which tyrosine is phosphorylated, we mutated Y225 or Y231 or both to alanine and transfected these three constructs into 293A cells. Surprisingly, Y225A mutation completely abolished phosphorylation of TIGIT (Figure 2b), but Y231A mutation was still phosphorylated as wild-type (WT) TIGIT did. As expected, both tyrosine mutations (Y225AY231A) failed to be phosphorylated (Figure 2b). We also transfected these three constructs into YTS cells and expression of TIGIT variants 
a

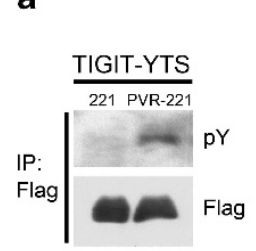

C

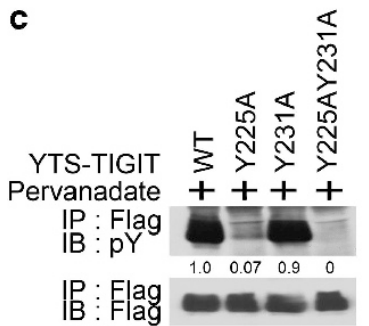

b

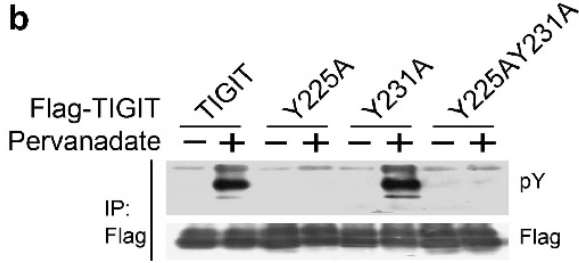

d

e
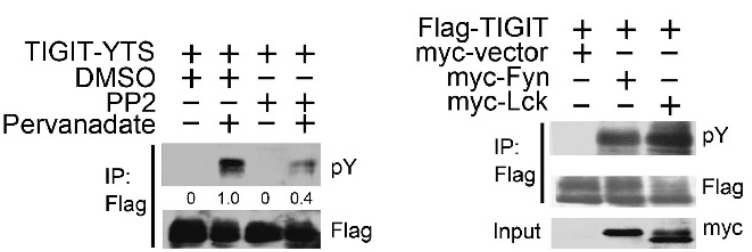

Figure 2 TIGIT is phosphorylated at its cytoplasmic tail after its ligation with PVR. (a) TIGIT is phosphorylated once engaged with PVR. TIGIT-YTS cells were incubated with 221 or PVR-221 cells at E:T ratio of $5: 1$ at $37^{\circ} \mathrm{C}$ for 5 min. Treated cells were lysed and Flag-tagged TIGIT was immunoprecipitated with anti-Flag beads followed by immunoblotting with anti-pY antibody. The same blot was stripped and reprobed with anti-Flag antibody. IP: immunoprecipitation; pY: phosphorylated tyrosine. (b) Tyr225A mutation abolishes tyrosine phosphorylation of TIGIT in 293A cells. Plasmids of $3 \times$ Flag-tagged TIGIT and its mutants (Y225A, Y231A or Y225AY231A) were transfected into $293 \mathrm{~A}$ cells for $48 \mathrm{~h}$ and treated with or without pervanadate for immunoblotting with anti-pY antibody. The same blot was stripped and reprobed with anti-Flag antibody. Y225A: Tyr225Ala mutation; Y231A: Tyr231Ala mutation; Y225AY231A: Tyr225AlaTyr231Ala mutation. (c) Tyr225A mutation abrogates tyrosine phosphorylation of TIGIT in YTS cells. Different mutants of TIGIT-YTS cells were treated with pervanadate for 10 min and detected as above. (d) PP2 can inhibit TIGIT phosphorylation. TIGIT-YTS cells were pretreated with a Src family inhibitor PP2 for $6 \mathrm{~h}$, and then treated with or without pervanadate for 10 min followed by immunoblotting as above. (e) Fyn and Lck can phosphorylate TIGIT. $3 \times$ Flag-TIGIT vector was co-transfected with myc-tagged Fyn or myc-tagged Lck vector into $293 \mathrm{~A}$ cells for $48 \mathrm{~h}$ and then lysed for immunoblotting. All above data are representative of at least three independent experiments. The numbers in Figure $2 \mathbf{c}$ and Figure $2 \mathbf{d}$ show relative amount of the indicated proteins

was at a similar level (Supplementary Figure S1A). By contrast, Y225A mutation could still be phosphorylated at a weak level in YTS cells (Figure 2c). The difference between phosphorylation of Y225A mutant in YTS and 293A cells might be attributed to different amounts of tyrosine kinases in these two cells lines. These data suggest that Y231 is also phosphorylated in YTS cells.

Most inhibitory receptors on NK cells are phosphorylated by the Src family tyrosine kinases, ${ }^{21,22}$ Fyn and Lck are highly expressed in NK cells. ${ }^{23}$ To detect whether Fyn and Lck are responsible for TIGIT phosphorylation, we treated TIGIT-YTS cells with PP2, an inhibitor that most efficiently inhibits these two Src family kinases. With treatment of pervanadate, an inhibitor of tyrosine phosphatases, TIGIT phosphorylation was detectable, otherwise its phosphorylation was undetectable (Figure 2d). We found that PP2 significantly reduced phosphorylation of TIGIT in the presence of pervanadate (Figure 2d). To further confirm Fyn and Lck can phosphorylate TIGIT, we coexpressed either myc-tagged Fyn or Lck with Flag-tagged TIGIT in 293A cells. Intriguingly, both Fyn and Lck were able to phosphorylate TIGIT (Figure 2e). These results indicate that TIGIT can be phosphorylated by Src family tyrosine kinases such as Fyn or Lck.

Phosphorylated TIGIT can recruit the adapter molecule Grb2 by its ITT-like motif. Y225 in TIGIT is located in a classical ITT-like motif, which exists in many receptors and can recruit Grb2, a widely expressed adapter molecule. 17,24 To determine whether TIGIT can recruit Grb2, we coexpressed Flag-tagged TIGIT and myc-tagged Grb2 in 293A cells. Only with pervanadate treatment, myc-Grb2 was coprecipitated with Flag-TIGIT (Figure 3a). The interaction of endogenous Grb2 with Flag-TIGIT was confirmed in TIGITYTS cells with pervanadate treatment (Figure $3 b$ ). These data suggest that Grb2 is recruited by phosphorylated TIGIT (pTIGIT). To verify the direct association between the pTIGIT and Grb2, we generated pTIGIT by coexpression of GSTTIGIT-His and Fyn in E. coli. Only pTIGIT could bind to recombinant Grb2 (rGrb2) (Figure 3c), whereas non-pTIGIT failed to precipitate rGrb2. As expected, Y225A or Y225AY231A mutant could not precipitate Grb2 in transfected 293A cells (Figure 3d). By contrast, Y231A mutant did not alter the binding efficiency compared with WT TIGIT. Similar results were obtained in transduced YTS cells and an in vitro pulldown assay (Figure $3 e$, Supplementary Figure S2A). However, Y225A mutant is still able to precipitate a little Grb2 in YTS cells (Figure 3e), suggesting that Grb2 is also required for the Y231 phosphorylation-mediated inhibition pathway. We further evaluated the interacting domain of Grb2 with pTIGIT. Interestingly, the N-terminal $\mathrm{SH} 3-\mathrm{SH} 2$ region was in charge of binding with pTIGIT (Supplementary Figure S2B), but the sole SH2 domain had no binding activity.

To check whether asparagine 227 (Asn227) in the ITT-like motif is also essential for Grb2 recruitment, we mutated Asn227 to glutamine (GIn) and transduced it to YTS cells to generate a TIGIT-N227Q stable cell line. We observed that TIGIT-N227Q mutant did not completely abolish the binding to Grb2 in YTS cells (Figure 3f), which implies that the ITT-like motif is the main domain involved in the transduction of the TIGIT inhibitory signal. However, TIGIT-N227Q was still tyrosine phosphorylated. To further confirm the above results, we coexpressed GST-TIGIT-N227Q-His mutant with Fyn in 


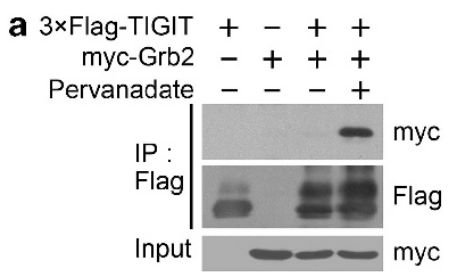

c

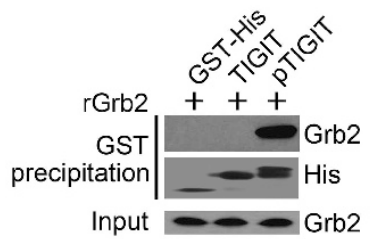

e
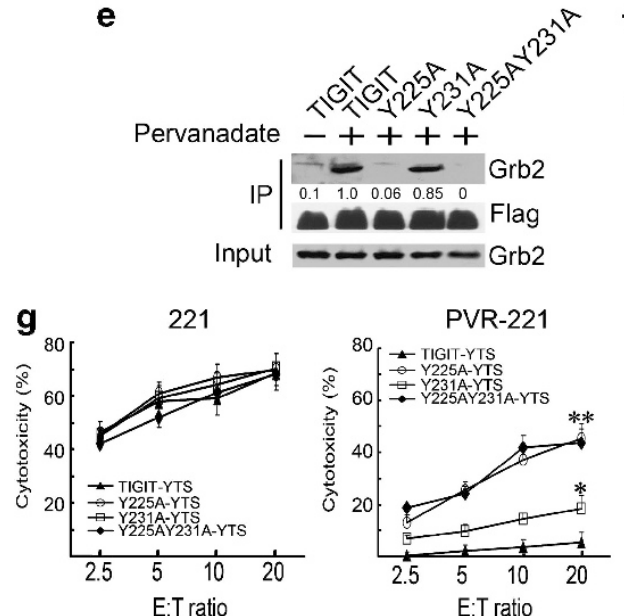

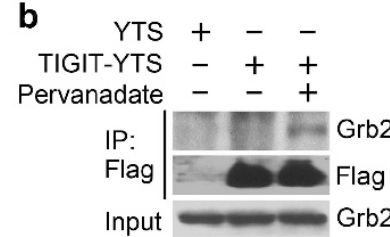

d
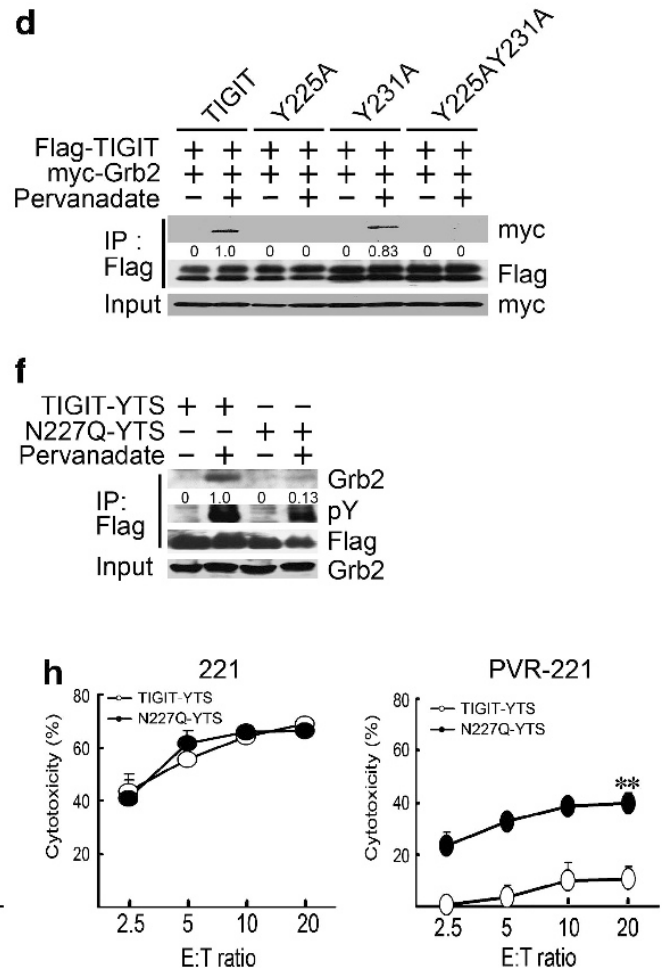

Figure 3 pTIGIT can recruit the adapter molecule Grb2 by its ITT-like motif. (a) pTIGIT associates with Grb2. Plasmids of $3 \times$ Flag-tagged TIGIT and myc-tagged Grb2 were co-transfected into $293 \mathrm{~A}$ cells for $48 \mathrm{~h}$. Transfected cells were lysed with or without pervanadate treatment and immunoprecipitated with anti-Flag beads followed by immunoblotting. myc-Grb2 in total lysates was probed for myc as a loading control. (b) pTIGIT can precipitate Grb2 in TIGIT-YTS cells. TIGIT-YTS or YTS cell lysates with or without pervanadate treatment were immunoprecipitated with anti-Flag beads and blotted with anti-Grb2 or anti-Flag antibody. Grb2 in the lysates was probed as a loading control. (c) pTIGIT can directly bind to rGrb2. Non-phosphorylated TIGIT (TIGIT) or pTIGIT was coincubated with recombinant His-Grb2 for a GST-pulldown assay. Precipitates were detected by immunoblotting with anti-Grb2 or anti-His antibody. Grb2 expression in the inputs served as a loading control. (d) Tyr225 mutation can not interact with Grb2 in 293A cells. Flag-tagged TIGIT mutant (Y225A, Y231A or Y225AY231A) and myc-Grb2 vectors were co-transfected into $293 \mathrm{~A}$ for $48 \mathrm{~h}$ and lysed with or without pervanadate treatment for immunoprecipitation. Myc-Grb2 expression in the inputs was used as a loading control. (e) Tyr225 mutation fails to bind Grb2 in YTS cells. Tyr225, Tyr231 or both mutated TIGIT-YTS cells with or without pervanadate treatment were lysed and immunoprecipitated with anti-Flag beads for immunoblotting. Grb2 in the inputs was used as a loading control. (f) Asn227 mutation abolishes the association of TIGIT with Grb2. TIGIT-YTS and TIGIT-N227Q-YTS (N227Q) cells were lysed with or without pervanadate treatment followed by immunoprecipitation. Grb2 in lysates was probed as a loading control. All above data represent at least three separate experiments. (g) Tyr225 mutation dramatically loses TIGIT/PVR-mediated killing inhibition. Tyr225 or Tyr231-mutated TIGIT-YTS cells were incubated with ${ }^{51} \mathrm{Cr}$-labeled 221 (left) or PVR-221 (right) target cells at the indicated E:T ratios followed by a ${ }^{51} \mathrm{Cr}$-release assay. (h) Asn227 mutation abrogates TIGIT/PVR-mediated killing inhibition against PVR-221 cells. TIGIT-N227Q-YTS (N227Q) or TIGIT-YTS cells were incubated with ${ }^{51} \mathrm{Cr}$-labeled PVR-221 or 221 cells as detected above. Data are representative of at least four independent experiments as means \pm S.D. ${ }^{\star} P<0.05$; ${ }^{*} P<0.01$. The numbers in Figures $3 \mathrm{~d}$, e and $\mathbf{f}$ show relative amount of the indicated proteins

E. coli. We found that GST-TIGIT-N227Q-His mutant was still phosphorylated (pN227Q) by Fyn (Supplementary Figure S2C). However, pN227Q could not precipitate Grb2 in YTS lysates (Supplementary Figure S2D), whereas PTIGIT could bind to Grb2. Taken together, both the phosphorylated Y225 and Asn227 of the ITT-like motif in TIGIT are essential for binding with Grb2. Moreover, Y225A mutant remarkably restored YTS-mediated cytotoxicity (Figure $3 \mathrm{~g}$ ), whereas Y231A mutant could only moderately restore YTS cytotoxicity. However, Y225A mutant and even the Y225AY231A double mutation did not completely rescued the killing capacity.
These results suggest that TIGIT may also mediate a tyrosine-independent inhibitory signal in NK cells, which remains to be further investigation. Additionally, TIGITN227Q-YTS cells abolished TIGIT/PVR-mediated inhibition for granule polarization (Supplementary Figure S3A) and declined cytotoxicity against PVR-221 cells (Figure 3h). Similar to Y225 mutants, the N227Q mutant failed to restore the cytotoxicity completely. Together, the ITT-like motif has a main role for TIGIT/PVR-mediated inhibitory signaling to attenuate NK effector function and the classical ITIM motif still has a role in TIGIT inhibition. 
Grb2 facilitates SHIP1 recruitment to TIGIT. Inhibitory receptors may recruit $\mathrm{SH} 2$-containing tyrosine phosphatases (SHP1 and SHP2) or SH2-containing inositol phosphatases (SHIP1 and SHIP2). ${ }^{25}$ We performed a co-immunoprecipitation (co-IP) assay and observed that PTIGIT could precipitate SHIP1 and SHP2 both in TIGIT-YTS cells (Figure 4a) and in primary NK cells (Figure 4b), but not SHP1. Moreover, pTIGIT could precipitate Grb2 in primary NK cells (Figure 4b). We observed that Y225A could bind a very little SHIP1 compared with WT TIGIT (Figure 4c), whereas Y225AY231A mutant failed to bind SHIP1. By contrast, while Y231A mutant still had the binding capacity with less amount than that of WT TIGIT. Consistent with these observations, Y231A-YTS cells still inhibited killing capacity against PVR-221 cells (Figure 3g), but Y225A-YTS cells showed much higher cytolysis against PVR-221 cells. These data indicate that Y225A mutation dramatically abolishes TIGIT/PVR-mediated killing inhibition, and Y225 has a major role in TIGIT/PVRmediated negative signaling. To test whether Asn227 in the ITT-like motif is also involved in SHIP1 accumulation, we generated phosphorylated GST-TIGIT-N227Q-His (pN227Q) and PTIGIT followed by a pulldown assay. Intriguingly, pN227Q almost lost capacity to bind SHIP1 (Figure 4d). Similar results were found in TIGIT-N227Q-transduced YTS cells (Figure 4e). We silenced Grb2 expression by RNA interference (RNAi) in TIGIT-YTS cells and performed a co-IP assay (Figure 4f). Grb2 silencing remarkably reduced the interaction of TIGIT with SHIP1. By contrast, SHP2 was unchangeable in Grb2 silencing and scramble control cells. Together, Grb2 can enhance SHIP1 recruitment to TIGIT.

TIGIT engagement inhibits PI3K and MAPK activation signaling in NK cells. Activation of both PI3K and MAPKs are essential for NK activity. ${ }^{26-28}$ SHIP1 is a key inhibitor of PI3K signaling, ${ }^{16,29}$ in which SHIP1 can hydrolyze $\mathrm{PI}(3,4,5) \mathrm{P}_{3}$ to inhibit accumulation and activation of plecktrin homology (PH) domain-containing kinases such as Akt, Btk and phospholipase $\mathrm{C}-\gamma$. The MAPK signaling cascade is a downstream PI3K effector in NK cells. ${ }^{26}$ Interestingly, TIGIT/PVR engagement prematurely terminated phosphorylation of Akt (Figure 5a), as well as of Erk and MEK kinases (Figure $5 b$ ). The latter kinases belong to effectors of MAPK signaling cascade. Cytosolic Erk phosphorylation was completely abolished when TIGIT was ligated with PVR by confocal microscopy (Figure $5 c$ ), whereas Erk was phosphorylated without PVR engagement. TIGIT-Y225A-YTS and TIGIT-Y231A-YTS showed inhibition of Erk phosphorylation with TIGIT/PVR engagement compared with without TIGIT/ PVR engagement (Figure 5d). Notably, TIGIT-Y225AY231AYTS double mutation did not completely abolish Erk phosphorylation. We incubated primary NK cells with PVR221 cells to determine a more physiological relevance. We found that TIGIT/PVR engagement could also prematurely terminate Erk phosphorylation in primary NK cells (Figure 5e). However, blockade of TIGIT signal significantly rescued Erk phosphorylation after TIGIT/PVR engagement (Figure 5f).

SHIP1 silencing opposes TIGIT/PVR-mediated inhibitory signaling and restores cytotoxicity of NK cells. To further determine whether SHIP1 affects NK cell function,
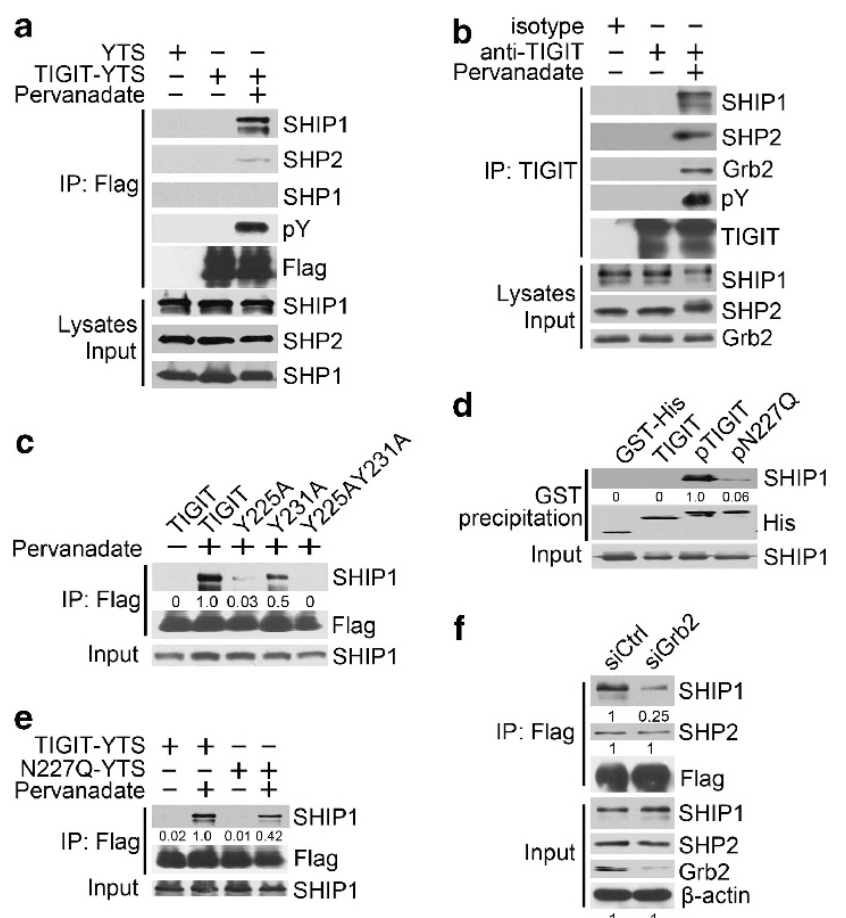

Figure 4 Grb2 facilitates SHIP1 recruitment to TIGIT. (a and b) pTIGIT mainly interacts with SHIP1. TIGIT-YTS (a) or primary NK cell (b) lysates with or without pervanadate treatment were immunoprecipitated by anti-Flag beads and immunoblotted with anti-SHIP1, anti-SHP2, anti-SHP1, anti-pY, or anti-Grb2 antibody. The same blots for testing $\mathrm{pY}$ were stripped and reprobed with anti-Flag antibody. (c) Tyr225 mutation fails to recruit SHIP1. TIGIT-YTS mutant (Y225A, Y231A or Y225AY231A) was transduced into YTS cells and lysed with or without pervanadate treatment for immunoprecipitation. SHIP1 in YTS lysates was used as a loading control. (d) Asn227 mutation can not associate with SHIP1. Recombinant non-phosphorylated TIGIT (TIGIT), pTIGIT, or phosphorylated TIGIT-Asn227GIn (pN227Q) were coincubated with YTS lysates and then immunoblotted with antiSHIP1 or anti-His antibody. SHIP1 in the inputs was used as a loading control. (e) Asn227 mutation of TIGIT remarkably diminishes its recruitment of SHIP1. N227Q-YTS or TIGIT-YTS cell lysates were immunoprecipitated by anti-Flag beads and immunoblotted with anti-SHIP1 or anti-Flag antibody. SHIP1 in lysates was used as a loading control. (f) Grb2 silencing dramatically reduces SHIP1 recruitment. TIGIT-YTS cells were transfected with siRNA duplexes either for Grb2 (siGrb2) or for scrambled siRNA control (siCtrl) for $48 \mathrm{~h}$. Lysates were immunoprecipitated with anti-Flag beads and immunoblotted for SHIP1, SHP2, Grb2 or Flag. SHIP1, SHP2 and $\beta$-actin in the inputs were used as loading controls. All above data are representative of at least three independent experiments. The numbers in Figures $4 \mathbf{c}-\mathbf{f}$ show relative amount of the indicated proteins

we silenced SHIP1 or SHP2 in TIGIT-YTS cells by RNAi (Figure 6a). SHIP1 knockdown could not suppress Erk phosphorylation after TIGIT/PVR ligation (Figure 6b), whereas SHP2 depletion still showed dramatic inhibition to Erk phosphorylation as the scramble siRNA control did. By contrast, without TIGIT ligation, Erk got phosphorylation in SHIP1 knockdown or SHP2 knockdown TIGIT-YTS cells when incubated with 721.221 cells (Figure 6b). Furthermore, SHIP1 knockdown or SHP2 knockdown did not change their cytolysis against 721.221 cells either (Figure 6c). However, SHIP1 silencing dramatically enhanced TIGIT-YTS-mediated cytotoxicity against PVR-221 cells (Figure 6c), whereas SHP2 knockdown had no such effect. Moreover, TIGITN227Q-YTS cells did not remarkably suppress Erk 
a

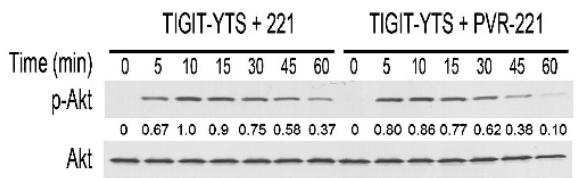

c CTB
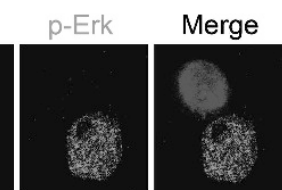

DIC
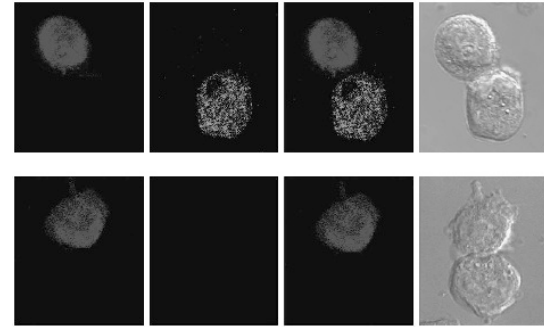

$\underset{+}{\text { TIGIT-YTS }}$ $\stackrel{+}{+}{ }^{+}$

e

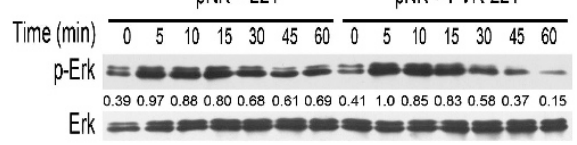

f

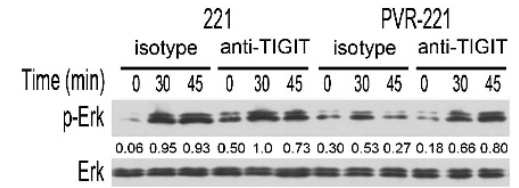

b

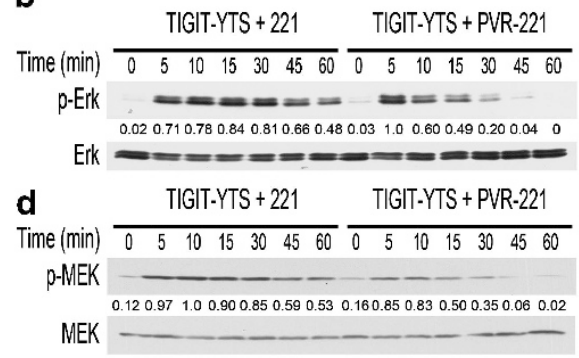

Y225A-YTS + $221 \quad$ Y225A-YTS + PVR-221

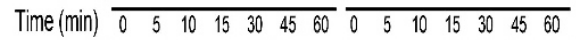
p-Erk $=-=--=-=-1=$ $0.650 .66 \quad 1.0 \quad 0.810 .800 .750 .140 .910 .700 .760 .670 .42 \quad 0.17$ Erk $==-===$ Y231A-YTS + $221 \quad$ Y231A-YTS + PVR-221

Time (min) \begin{tabular}{llllllllllllll}
\hline & 5 & 10 & 15 & 30 & 45 & 60 & 0 & 5 & 10 & 15 & 30 & 45 & 60
\end{tabular} p-Erk - - - - - - - - 0.071 .00 .760 .740 .690 .630 .600 .200 .970 .690 .650 .340 .030

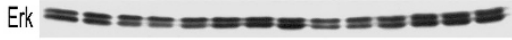
Y225AY231A-YTS + $221 \quad$ Y225AY231A-YTS + PVR-221

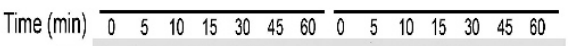
p-Erk $\quad-=-=-=-=$ $\begin{array}{llllllllllllll}0 & 0.200 .59 & 0.87 & 1.0 & 0.56 & 0.38 & 0.02 & 0.31 & 0.64 & 0.71 & 0.810 .44 & 0.06\end{array}$

Figure 5 TIGIT engagement prematurely terminates PI3K and MAPK activation signaling in YTS cells. (a and $\mathbf{b})$ TIGIT/PVR ligation prematurely terminates phosphorylation of Akt or of Erk and MEK. TIGIT-YTS cells were stimulated with 221 or PVR-221 cells followed by immunoblotting with anti-p-Akt antibody (a, upper) and the same blot was reprobed with anti-Akt antibody (a, lower). p-Erk (b, upper panel) and p-MEK (b, lower panel) were detected as above. (c) TIGIT/PVR engagement inhibits Erk phosphorylation by confocal microscopy. TIGIT-YTS cells were incubated with CellTracker Blue-labeled 221 (upper) or PVR-221 (lower) cells for 45 min and stained for $p$-Erk followed by confocal microscopy. Blue fluorescence for target cells is shown in the first lane, red for $p$-Erk in the second lane, the merged image in the third lane, differential interference control (DIC) in the last lane. CTB: CellTracker Blue. (d) Tyr225 mutation disrupts TIGIT/PVR-mediated premature termination of Erk phosphorylation. TIGITY225A-YTS (Y225A-YTS), TIGIT-Y231A-YTS (Y231A-YTS), TIGIT-Y225AY231A-YTS (Y225AY231A-YTS) cells were incubated with PVR-221 or 221 cells and immunoblotted with anti-p-Erk or anti-Erk antibody. (e) TIGIT/PVR ligation attenuates phosphorylation of Erk in primary NK cells. Human primary NK cells were incubated with PVR-221 or 221 cells and immunoblotted with anti-p-Erk or anti-Erk antibody. (f) Blockade of TIGIT disrupts the premature termination of Erk phosphorylation. TIGIT-YTS cells were preincubated with anti-TIGIT ( $40 \mu \mathrm{g} / \mathrm{ml})$ or isotype control $\mathrm{mlgG}$ for $30 \mathrm{~min}$ on ice, then were incubated with PVR-221 or 221 cells for the indicated times. All above data represent at least three separate experiments. The numbers in Figures $5 \mathbf{a}$ and $\mathbf{b}$ and Figures $5 \mathbf{d}-\mathbf{f}$ show relative amount of the indicated proteins. The color representation of this figure is available at the Cell Death and Differentiation Journal online

phosphorylation after incubated with PVR-221 cells compared with 721.221 cells (Supplementary Figure S3B).

\section{Discussion}

TIGIT was recently identified as an inhibitory receptor both on T cells and NK cells. ${ }^{10-12}$ However, it is not clear about how TIGIT mediates an inhibitory signaling pathway in T and NK cells. Herein we demonstrate that TIGIT/PVR engagement disrupts granule polarization leading to loss of killing activity of NK cells. The ITT-like motif of TIGIT has a major role in its negative signaling. After TIGIT/PVR ligation, the ITT-like motif is mainly phosphorylated at Tyr225 and binds to cytosolic adapter Grb2, which can recruit SHIP1 to suppress PI3K and MAPK signaling cascade to downregulate NK cell activity. By contrast, the classical ITIM motif has a minor role in TIGIT inhibitory signal.

Effector functions of NK cells depend on a balanced modulation of various activating and inhibitory receptors. ${ }^{30}$ Inhibitory receptors share a common ITIM motif in their cytoplasmic tails, whose tyrosine phosphorylation is the first step in the inhibitory signaling pathway. ${ }^{31}$ Once phosphorylated,
ITIMs recruit mostly the protein tyrosine phosphatases SHP1 and SHP2. ${ }^{8}$ Here we showed that TIGIT/PVR engagement initiates an inhibitory signaling mainly through its ITT-like motif. Tyr231 mutation (Y231A) weakly affected the tyrosine phosphorylation of TIGIT, whereas Tyr225 mutation almost lost its phosphorylation. Moreover, Tyr231 mutation of TIGIT still showed much weaker killing capacity against PVR-221 cells, whereas Tyr225 mutation appeared to have much higher cytotoxicity. Our findings suggest that Tyr225 has a major role in TIGIT/PVR-mediated negative signaling, whereas Tyr231 exerts a minor inhibitory role. Our results are distinct from a recent report $^{11}$ in which they found Tyr231 mutation or truncation of the ITIM lost TIGIT-mediated inhibitory cytolysis. However, they did not precipitate SHP1 with TIGIT. They also proposed that TIGIT may mediate a different inhibitory pathway from other NK inhibitory receptors.

Surprisingly, we found that NK cell TIGIT can associate with the cytosolic adapter Grb2 mainly through its ITT-like phosphorylation motif. It was shown that the ITT phosphorylation motif of membrane-bound IgG and IgE induces ITAMinduced signaling and B-cell proliferation. ${ }^{18}$ The ITT-like motif has also been identified in costimulatory receptors on $\mathrm{T}$ and 
a

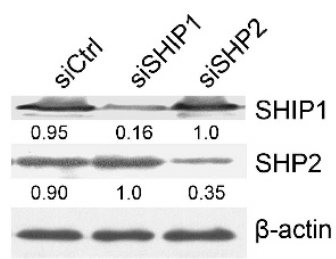

b

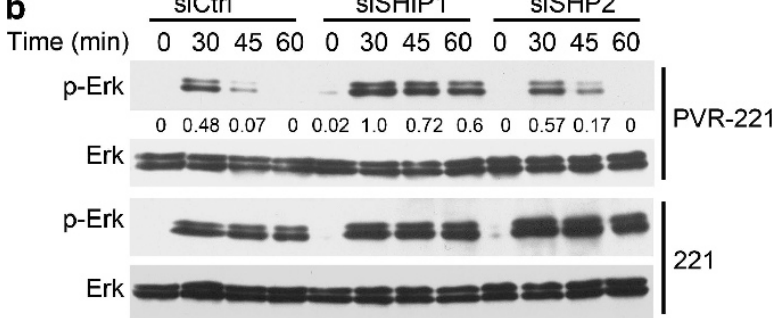

C

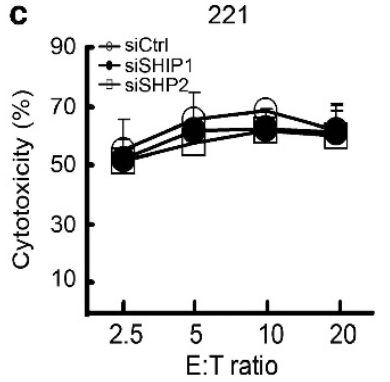

PVR-221

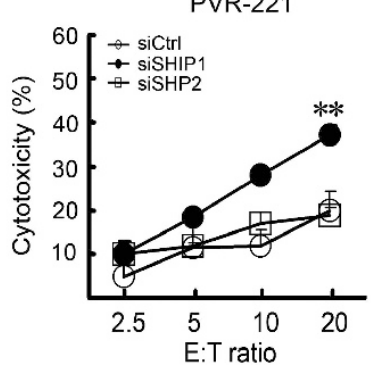

Figure 6 SHIP1 silencing opposes TIGIT/PVR-mediated inhibitory signaling and restores cytotoxicity of YTS cells. (a) SHIP1 expression was silenced in TIGIT-YTS cells. TIGIT-YTS cells were transfected with siRNA duplexes for SHIP1 (siSHIP1), SHP2 (siSHP2) or scrambled siRNA control (siCtrl) for $48 \mathrm{~h}$ and confirmed by immunoblotting. $\beta$-Actin was probed as a negative control. (b) SHIP1 depletion rescues Erk phosphorylation. SHIP1- or SHP2-silenced TIGIT-YTS cells were incubated with PVR-221 or 221 cells and detected for Erk phosphorylation. (c) SHIP1 knockdown restores TIGIT-YTS-mediated cytolysis against PVR-221 cells. SHIP1- or SHP2-silenced TIGIT-YTS cells were incubated with ${ }^{51} \mathrm{Cr}$-labeled PVR-221 or 221 cells at different $\mathrm{E}$ :T ratios for $^{51} \mathrm{Cr}$-release assay. Data are representative of at least four independent experiments as means \pm S.D. ${ }^{* *} P<0.01$. The numbers in Figures $6 \mathbf{a}$ and $\mathbf{b}$ show relative amount of the indicated proteins

NK cells. ${ }^{13}$ However, it is unclear how ITT-like motif induces an inhibitory signaling pathway in immunological responses. Here, we found that the ITT-like motif can be phosphorylated by Src family kinase Fyn or Lck. Tyr225 phosphorylation and Asn227 are critical for recruitment of Grb2. After its phosphorylation, TIGIT can accumulate $\mathrm{SH} 2$ domain-containing inositol phosphatase SHIP1, leading to inhibitory signaling in NK cells. Our findings are in agreement with previous results. ${ }^{13}$ Both SHIP and SHP2 require a single phosphorylated tyrosine to interact, but SHP1 needs the ITIM-like double phosphorylated tyrosines for association.

SHIP-knockout mice cause severe immune deficiency owing to aberrations in the expression of NK receptors or downstream signaling. ${ }^{32,33}$ SHIP can convert $\mathrm{PI}(3,4,5) \mathrm{P}_{3}$ to $\mathrm{PI}(3,4) \mathrm{P}_{2}$, whereas $\mathrm{PI}(3,4,5) \mathrm{P}_{3}$ is a product of $\mathrm{PI} 3 \mathrm{~K}$. $\mathrm{PI}(3,4,5) \mathrm{P}_{3}$ is accumulated in the plasma membrane to provide recruitment sites for signaling proteins containing $\mathrm{PH}$ domains, including Akt, PDK1, Grp1, DAPP and Tec family kinases. ${ }^{16}$ Hydrolysis of $\mathrm{PI}(3,4,5) \mathrm{P}_{3}$ blocks recruitment of $\mathrm{PH}$ domain-containing kinases to the plasma membrane to inhibit PI3K signaling, resulting in inactivation of some downstream PI3K effectors. These downstream effectors include Erk, JNK/SAPK, p38 MAPK and major transcription factors such as NF- $\kappa \mathrm{B}$ and NFAT, ${ }^{34,35}$ which modulate cell survival, proliferation and effector functions. As we observed, SHIP1 recruitment by TIGIT prematurely terminated activation of Akt, Erk and MEK, leading to inhibition of granule polarization as well as cytotoxicity of NK cells.

NK effector functions are well orchestrated by a wide array of NK receptor repertoire. TIGIT shares coligands PVR (CD155) and PVRL2 (CD112) with activating receptor CD226 (DNAM-1), which elicits activation signaling and promotes NK cytotoxicity. ${ }^{36}$ The hallmark of ITT-like motifs is their functional reliance on simultaneous ITAM activation. ${ }^{13}$ After activated NK cells eradicate virus-infected or -transformed cells, they are simultaneously required for inhibitory signaling to terminate NK cell execution for preventing killing from self normal cells. Two recent reports showed that TIGIT/ PVR binding is dominant over that of CD226-PVR, ${ }^{10,11}$ which implies that TIGIT/PVR-mediated inhibitory signaling is critical for the regulation of NK cell function. It was reported that PVR and PVRL2 are highly expressed on various tumors. ${ }^{36,37}$ TIGIT/PVR-mediated inhibitory signaling may contribute to tumorigenesis. Thus, it is of great significance to elucidate the molecular mechanisms of TIGIT/CD226-PVR/PVRL2 signaling pathways for NK and T-cell functions to design new strategy for tumor therapy.

\section{Materials and Methods}

Cell lines and reagents. YTS and 721.221 cells were grown in RPMI 1640 as described ${ }^{38}$ and $293 \mathrm{~A}$ cells were grown in DMEM medium as described, ${ }^{39}$ both of which were supplemented with $10 \%$ fetal calf serum (Gibco, Carlsbad, CA, USA), $50 \mu \mathrm{M} \beta$-mercaptoethanol, $100 \mathrm{units} / \mathrm{ml}$ penicillin and $100 \mu \mathrm{g} / \mathrm{ml}$ streptomycin. YTS cells transfected with Flag-TIGIT and its mutants, and 721.221 cells transfected with PVR were cultured with additional $0.5 \mu \mathrm{g} / \mathrm{ml}$ of puromycin. CellTracker Blue CMAC was from Invitrogen (Carlsbad, CA, USA), Hoechst 33342 was from Sigma-Aldrich (St. Louis, MO, USA) and CFSE was purchased from Molecular Probes (Carlsbad, CA, USA).

Isolation of primary NK cells. Peripheral blood from healthy donors was obtained from Beijing Blood Center (Beijing, China) and PBMCs were purified by Ficoll gradient centrifugation. NK cells were further purified by negative selection using the human NK cell isolation Kit (Miltenyi Biotec, Bergisch Gladbach, Germany). The purity of NK cells was above $90 \%$ by FACS analysis. Purified NK cells were cultured in medium containing 200 units $/ \mathrm{ml} \mathrm{IL-2.}$

Antibodies. Antibodies used were from as follows: anti-human TIGIT PE (eBioscience, San Diego, CA, USA); anti-PVR; Perforin antibody (ab47225) from 
Abcam (Cambridge, UK); anti-phosphotyrosine 4G10 Platinum 05-1050X (Millipore, Billerica, MA, USA); Phospho-Tyrosine (pTyr-100), SHIP1 (C4099) Rabbit mAb, SHP2 antibody, SHP1(C14H6) Rabbit mAb, phospho-p44/42 MAPK (Erk1/2) (Thr202/Tyr294), p44/42 MAPK (Erk1/2) (137F5) Rabbit mAb, Phospho-Akt (Ser473), Akt antibody, Phospho-MEK1/2 (Ser217/221) (41G9) Rabbit mAb, MEK1/2 antibody and $\beta$-tubulin (9F3) Rabbit mAb (Cell Signaling, Danvers, MA, USA); Monoclonal ANTI-Flag M2 antibody and monoclonal ANTI-polyhistidine clone His-1 (Sigma-Aldrich); A-c-myc, GST(B14)(sc-138) and Grb2 (C7) (sc-8034) (Santa Cruz, Santa Cruz, CA, USA); anti-DDDDK-Tag (MBL, Woburn, MA, USA); $\mathrm{PE}$-goat anti-mouse IgG (Sanjian, Tianjin, China).

Plasmid construction. The human full-length DNA for TIGIT was subcloned into $3 \times$ Flag-CMV-14 vector (Sigma-Aldrich). Human full-length Grb2 was inserted into pcDNA4/myc-HisB vector (Invitrogen). myc-Fyn and myc-Lck plasmids were kind gift from Dr. Byung-Chul Oh (Gachon University of Medicine and Science, Korea). Fyn DNA was amplified from myc-Fyn vector and subcloned into pACYCDuet-1 (Novagen, Madison, WI, USA) for E. coli expression. The cytoplasmic tail of human TIGIT (493-735) (WT) and its mutants were subcloned into PGEX6P-1 (GE Healthcare, Waukesha, WI, USA). Full-length and its truncated fragments (13-474 and 177-474) of human Grb2 were cloned into pET28a. All constructs were confirmed by DNA sequencing.

Lentivirus package and infection. pSIN-EF2-puro vector with Flag-TIGIT or HA-PVR was co-transfected with psPAX2 and pMD2.G plasmids into 293T cells. After 48 and $72 \mathrm{~h}$, the viruses were collected and stored in $-80^{\circ} \mathrm{C}$ as described. ${ }^{4}$ For infection, YTS or 221 cells were incubated with the above obtained lentivirus at $37^{\circ} \mathrm{C}$ for $20 \mathrm{~min}$.

Flow cytometry analysis. YTS-TIGIT cells or 221-PVR cells were stained with anti-TIGIT or anti-PVR antibody for $20 \mathrm{~min}$ at $4{ }^{\circ} \mathrm{C}$. After complete washing, treated cells were added with PE-conjugated goat anti-mouse IgG for $15 \mathrm{~min}$ followed by flow cytometry.

RNA interference. siRNA duplexes for human Grb2, SHIP1 and SHP2 were synthesized by GenePharma (Shanghai, China). The siRNA sequences are as follows: Grb2: 5'-UCAGCCAAUUUGUCUCCUATT-3'; SHIP1: 5'-GCCCAUAU CACCCAAGAAGUUTT-3'; and SHP2: 5'-GGGCCAGAGCAGUCAGUAATT-3'. For each siRNA, it was scrambled for a negative control. siRNAs were transfected into YTS cells with Program 0-017 from the manufacturer's introductions of the Nucleofector Kit R (Lonza, Basel, Switzerland).

Laser scanning confocal microscopy. 221 or PVR-221 cells were prelabeled with CellTracker Blue for $20 \mathrm{~min}$. Then they were incubated with YTS or TIGIT-YTS cells for different times. Mixed cells were loaded on poly-lysine pretreated cover glass. Cells were permeabilized with $0.5 \%$ NP40 or Digitonin (Sigma-Aldrich) after a $30 \mathrm{~min}$ fixation in $4 \%$ paraformaldehyde. Then cells were stained with anti-p-Erk, anti-perforin, anti- $\beta$-tubulin or anti-Flag antibody and then with the fluorescence-labeled second antibody before confocal microscopy

Immunoprecipitation and immunoblotting. Treated cells were centrifuged and their pellets were lysed in a lysis buffer $(20 \mathrm{mM}$ Tris- $\mathrm{HCl}(\mathrm{pH} 7.4)$, $150 \mathrm{mM} \mathrm{NaCl}, 1 \mathrm{mM} \mathrm{Na} \mathrm{EDDTA}_{2}$ (pH 5.0), 0.5\% (v/v) NP40, $1 \mathrm{mM} \mathrm{Na}_{3} \mathrm{VO}_{4}, 10 \%$ (v/v) glycerol and protease inhibitors) for $20 \mathrm{~min}$. Supernatants were incubated with antiFlag beads overnight and boiled in $1 \times$ SDS loading buffer for immunoblotting.

Protein expression and pulldown assay. PGEX6P-1-TIGIT-His was expressed or coexpressed with pACYCDuet-1-Fyn in E. coli strain BL21, respectively. PGEX6P-1-TIGIT-His mutants with a C-terminal $6 \times$ His tag were also coexpressed with pACYCDuet-1-Fyn in E. coli strain BL21. His-Grb2 was expressed with pET28-Grb2 plasmids in $E$. coli strain Rosseta. For pulldown assay, rGrb2 was incubated with equal amount of GST-His-tagged TIGIT for $2 \mathrm{~h}$ at $4{ }^{\circ} \mathrm{C}$ and then mixed with GST beads followed by immunoblotting. For pulldown for YTS cells, YTS cell lysates ( $10^{7}$ equivalents) were incubated with equal amount of recombinant protein and analyzed as above.

Conjugation assay. YTS or TIGIT-YTS cells were stained with Hoechst and 221 or PVR-221 cells were dyed with CFSE. Then effector cells were incubated with target cells at an E:T ratio of $1: 2$. Mixed cells were spun down at a minimal speed for $1 \mathrm{~min}$ and incubated at $37^{\circ} \mathrm{C}$ for the indicated time points. Treated cells were vortexed at high speed for $3 \mathrm{~s}$ and added ice-cold $0.5 \%$ paraformaldehyde immediately followed by flow cytometry.

Cytotoxicity assay. YTS or TIGIT-YTS cells were incubated with ${ }^{51} \mathrm{Cr}$-labeled 221 or 221-PVR cells at different effecter: target (E:T) ratios followed by a standard ${ }^{51} \mathrm{Cr}$-release assay, and specific cytotoxicity was calculated as described. ${ }^{40}$

\section{Conflict of Interest}

The authors declare no conflict of interest.

Acknowledgements. We thank Drs. André Veillette and Geng Zhang for critical discussion; Dr. Byung-Chul Oh for providing plasmids; Dr. Jane L Grogan for providing some antibodies. We also thank Junying Jia, Chunchun Liu and Yan Teng for technical assistance and Shuo Wang, Li Wang, Chao Zhong and Pengyan Xia for helpful discussions. This work was supported by the National Natural Science Foundation of China (30830030, 31170837, 30972676), the grants from the Ministry of Science and Technology China (2010CB911902) and from the Chinese Academy of Sciences (XDA01010407).

\section{Author contributions}

$\mathrm{SL}$ and $\mathrm{HZ}$ designed and performed research, analyzed data and wrote the manuscript; $\mathrm{ML}, \mathrm{DH}$ and $\mathrm{CL}$ performed some experiments; $\mathrm{BG}$ and $\mathrm{BJ}$ provided vectors and cell lines and analyzed the data; ZF initiated the research, analyzed the data and wrote the manuscript.

1. Vivier E, Tomasello E, Baratin M, Walzer T, Ugolini S. Functions of natural killer cells. Nat Immunol 2008; 9: 503-510.

2. Fan Z, Yu P, Wang Y, Fu ML, Liu W, Sun Y et al. NK-cell activation by LIGHT triggers tumor-specific CD8 + T-cell immunity to reject established tumors. Blood 2006; 107: 1342-1351

3. Zhong C, Li C, Wang X, Toyoda T, Gao G, Fan Z. Granzyme K inhibits replication of influenza virus through cleaving the nuclear transport complex importin alpha1/beta dimer of infected host cells. Cell Death Differ 2011; 19: 882-890

4. Tang $\mathrm{H}$, Li C, Wang L, Zhang H, Fan Z. Granzyme $\mathrm{H}$ of cytotoxic lymphocytes is required for clearance of the hepatitis $B$ virus through cleavage of the hepatitis $B$ virus $X$ protein. J Immunol 2012; 188: 824-831.

5. Orr MT, Lanier LL. Natural killer cell education and tolerance. Cell 2010; 142: 847-856.

6. Hoglund P, Brodin P. Current perspectives of natural killer cell education by MHC class I molecules. Nat Rev Immunol 2010; 10: 724-734.

7. Elliott JM, Yokoyama WM. Unifying concepts of MHC-dependent natural killer cell education. Trends Immunol 2011; 32: 364-372.

8. WatzI C, Long EO. Signal transduction during activation and inhibition of natural killer cells. Curr Protoc Immunol 2010: Chapter 11: unit 11.9B.

9. Kumar V, McNerney ME. A new self: MHC-class-I-independent natural-killer-cell selftolerance. Nat Rev Immunol 2005; 5: 363-374.

10. Yu X, Harden K, Gonzalez LC, Francesco M, Chiang E, Irving B et al. The surface protein TIGIT suppresses $T$ cell activation by promoting the generation of mature immunoregulatory dendritic cells. Nat Immunol 2009; 10: 48-57.

11. Stanietsky N, Simic H, Arapovic J, Toporik A, Levy O, Novik A et al. The interaction of TIGIT with PVR and PVRL2 inhibits human NK cell cytotoxicity. Proc Natl Acad Sci USA 2009; 106: 17858-17863.

12. Boles KS, Vermi W, Facchetti F, Fuchs A, Wilson TJ, Diacovo TG et al. A novel molecular interaction for the adhesion of follicular CD4 T cells to follicular DC. Eur J Immunol 2009; 39: 695-703.

13. Engels N, Wienands J. The signaling tool box for tyrosine-based costimulation of Iymphocytes. Curr Opin Immunol 2011; 23: 324-329.

14. Joller N, Hafler JP, Brynedal B, Kassam N, Spoerl S, Levin SD et al. Cutting edge: TIGIT has T cell-intrinsic inhibitory functions. J Immunol 2011; 186: 1338-1342.

15. Levin SD, Taft DW, Brandt CS, Bucher C, Howard ED, Chadwick EM et al. Vstm3 is a member of the CD28 family and an important modulator of T-cell function. Eur J Immunol 2011; 41: 902-915

16. Kerr WG, Colucci F. Inositol phospholipid signaling and the biology of natural killer cells. $\mathrm{J}$ Innate Immun 2011; 3: 249-257.

17. Neumann K, Oellerich T, Urlaub H, Wienands J. The B-lymphoid Grb2 interaction code. Immunol Rev 2009; 232: 135-149.

18. Engels N, Konig LM, Heemann C, Lutz J, Tsubata T, Griep S et al. Recruitment of the cytoplasmic adaptor Grb2 to surface $\mathrm{lgG}$ and $\mathrm{lgE}$ provides antigen receptor-intrinsic costimulation to class-switched B cells. Nat Immunol 2009; 10: 1018-1025. 
19. Sakisaka T, Ikeda W, Ogita H, Fujita N, Takai Y. The roles of nectins in cell adhesions: cooperation with other cell adhesion molecules and growth factor receptors. Curr Opin Cell Biol 2007; 19: 593-602.

20. Takai Y, Miyoshi J, Ikeda W, Ogita H. Nectins and nectin-like molecules: roles in contact inhibition of cell movement and proliferation. Nat Rev Mol Cell Biol 2008; 9: 603-615.

21. Binstadt BA, Brumbaugh KM, Dick CJ, Scharenberg AM, Williams BL, Colonna M et al. Sequential involvement of Lck and SHP-1 with MHC-recognizing receptors on NK cells inhibits FcR-initiated tyrosine kinase activation. Immunity 1996; 5: 629-638.

22. Marti F, Xu CW, Selvakumar A, Brent R, Dupont B, King PD. LCK-phosphorylated human killer cell-inhibitory receptors recruit and activate phosphatidylinositol 3-kinase. Proc Natl Acad Sci USA 1998; 95: 11810-11815.

23. Lowin-Kropf B, Kunz B, Schneider P, Held W. A role for the src family kinase Fyn in NK cell activation and the formation of the repertoire of Ly49 receptors. Eur J Immunol 2002; 32: 773-782.

24. Otipoby KL, Draves KE, Clark EA. CD22 regulates B cell receptor-mediated signals via two domains that independently recruit Grb2 and SHP-1. J Biol Chem 2001; 276: 44315-44322.

25. Long EO. Negative signaling by inhibitory receptors: the NK cell paradigm. Immunol Rev 2008; 224: 70-84.

26. Jiang $\mathrm{K}$, Zhong B, Gilvary DL, Corliss BC, Hong-Geller E, Wei S et al. Pivotal role of phosphoinositide-3 kinase in regulation of cytotoxicity in natural killer cells. Nat Immunol 2000; 1: 419-425.

27. Li C, Ge B, Nicotra M, Stern JN, Kopcow HD, Chen X et al. JNK MAP kinase activation is required for $\mathrm{MTOC}$ and granule polarization in NKG2D-mediated NK cell cytotoxicity. Proc Natl Acad Sci USA 2008; 105: 3017-3022.

28. Chen X, Trivedi PP, Ge B, Krzewski K, Strominger JL, Many NK. Cell receptors activate ERK2 and JNK1 to trigger microtubule organizing center and granule polarization and cytotoxicity. Proc Natl Acad Sci USA 2007; 104: 6329-6334.

29. Ong CJ, Ming-Lum A, Nodwell M, Ghanipour A, Yang L, Williams DE et al. Small-molecule agonists of SHIP1 inhibit the phosphoinositide 3-kinase pathway in hematopoietic cells. Blood 2007; 110: 1942-1949.
30. Johansson S, Berg L, Hall H, Hoglund P. NK cells: elusive players in autoimmunity. Trends Immunol 2005; 26: 613-618.

31. Lanier LL. Up on the tightrope: natural killer cell activation and inhibition. Nat Immunol 2008; 9: 495-502.

32. Wang JW, Howson JM, Ghansah T, Desponts C, Ninos JM, May SL et al. Influence of SHIP on the NK repertoire and allogeneic bone marrow transplantation. Science 2002; 295: 2094-2097.

33. Wahle JA, Paraiso KH, Kendig RD, Lawrence HR, Chen L, Wu J et al. Inappropriate recruitment and activity by the Src homology region 2 domain-containing phosphatase 1 (SHP1) is responsible for receptor dominance in the SHIP-deficient NK cell. $J$ Immunol 2007; 179: 8009-8015.

34. Robson JD, Davidson D, Veillette A. Inhibition of the Jun N-terminal protein kinase pathway by SHIP-1, a lipid phosphatase that interacts with the adaptor molecule Dok-3. Mol Cell Biol 2004; 24: 2332-2343.

35. Ono M, Okada H, Bolland S, Yanagi S, Kurosaki T, Ravetch JV. Deletion of SHIP or SHP-1 reveals two distinct pathways for inhibitory signaling. Cell 1997; 90: 293-301.

36. Bottino C, Castriconi R, Pende D, Rivera P, Nanni M, Carnemolla B et al. Identification of PVR (CD155) and Nectin-2 (CD112) as cell surface ligands for the human DNAM-1 (CD226) activating molecule. J Exp Med 2003; 198: 557-567.

37. Sloan KE, Eustace BK, Stewart JK, Zehetmeier C, Torella C, Simeone M et al. CD155/PVR plays a key role in cell motility during tumor cell invasion and migration. BMC Cancer 2004; 4: 73.

38. Yu MC, Su LL, Zou L, Liu Y, Wu N, Kong L et al. An essential function for beta-arrestin 2 in the inhibitory signaling of natural killer cells. Nat Immunol 2008; 9: 898-907.

39. Hu D, Liu S, Shi L, Li C, Wu L, Fan Z. Cleavage of survivin by Granzyme M triggers degradation of the survivin-X-linked inhibitor of apoptosis protein (XIAP) complex to free caspase activity leading to cytolysis of target tumor cells. J Biol Chem 2010; 285 18326-18335.

40. Fan Z, Beresford PJ, Zhang D, Xu Z, Novina CD, Yoshida A et al. Cleaving the oxidative repair protein Ape1 enhances cell death mediated by granzyme A. Nat Immunol 2003; 4 145-153.

Supplementary Information accompanies the paper on Cell Death and Differentiation website (http://www.nature.com/cdd) 